\title{
Aerodynamic and Structural Evaluation of Horizontal Archimedes Spiral Wind Turbine
}

\author{
Arman Safdari and Kyung Chun Kim
}

\begin{abstract}
Aerodynamic characteristics of small-scale of Archimedes spiral wind turbine blade are presented in this paper. Numerical simulation for aerodynamic performance of the blade was carried out for different configuration of inlet velocity. Numerical approaches on the prediction of aerodynamic characteristics of the blade were performed by using XFlow which has been written based on lattice Boltzmann method. Wall-Adapting Local Eddy-viscosity (WALE) model has been applied as is has a good properties near and far from solid body and wall for both laminar and turbulent flows. Particle Image Velocity (PIV) has been used to prove the obtained results of numerical simulation and investigate the aerodynamic physiognomies of the spiral wind turbine. In order to verify the numerical analysis velocity behavior around the blade are captured and compared with experimental results. The prediction of velocity outlines by using XFlow is in a good agreement with the trajectory and greatness of tip vortices engendered by the Archimedes spiral wind turbine blade from experimental results.
\end{abstract}

Index Terms - Lattice boltzmann method, wind turbine, archimedes spiral, wall-adapting local eddy model, WALE.

\section{INTRODUCTION}

Wind is a clean source of renewable energy that produces no air or water pollution. And since the wind is free, operational costs are nearly zero once a turbine is erected. Besides, quantity production and technology improvements are making turbines cheaper. Among all renewable resources, wind energy has been proven to be a relatively matured technology and has great potential in commercialization and the production of large quantities. The main application of wind power is generation of electricity from a power system network integrates transmission grids [1], [2]. Since big-scale wind turbines need large grid-connected wind farms, small-size wind turbine has been designed in fields such as mobile communication base stations, city road lighting, offshore aquaculture and sea water purification in several countries [3].

In general, small wind turbines are classified in two types of wind turbine HAWT (Horizontal axis wind turbine) and

Manuscript received November 25, 2013; revised January 15, 2014. This study was supported by the National Research Foundation of Korea (NRF) grant funded by the Korea government (MSIP) through GCRC-SOP (No. 2011-0030663) and by the Korea Institute of Energy Technology Evaluation and Planning (KETEP) grant funded by MSIP (No. 20113030020010-11-2-100).

A. Safdari is with the School of Mechanical Engineering, Pusan National University, Busan 609-735, Republic of Korea (e-mail: safdari.arman@pusan.ac.kr).

K. C. Kim is with the School of Mechanical Engineering, Pusan National University, Busan 609-735, Republic of Korea (e-mail: kckim@pusan.ac.kr).
VAWT (Vertical axis wind turbine) [4], [5]. Archimedes spiral wind turbine, is one of the HAWT, however, there is marked contrast between new wind turbine design and traditional HAWT models. The spiral allowed better measurement of a circle's circumference and thus its area. However, this spiral was soon proved inadequate when Archimedes went on to determine a more accurate value of $\mathrm{Pi}$ that created an easier way of measuring the area of a circle [6].

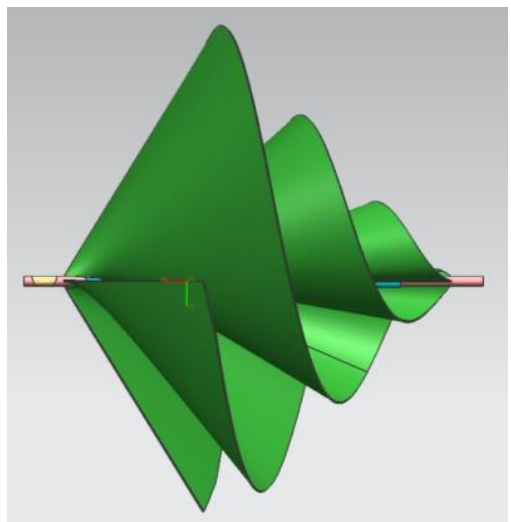

(a) A snapshot of the spiral wind turbine blade at X-Y plane.

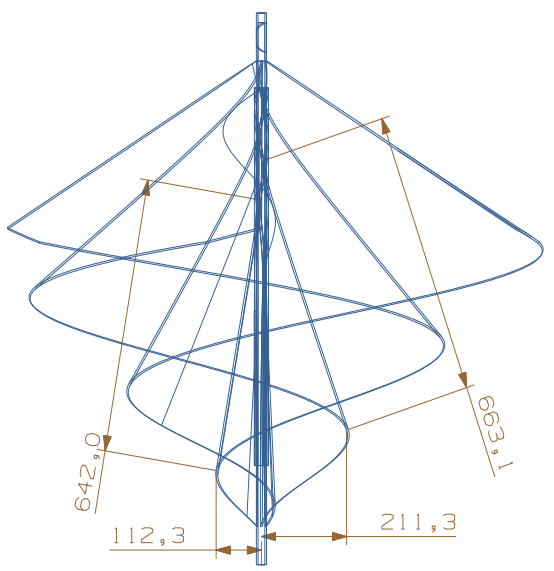

(b) Geometry of $0.5 \mathrm{KW}$ Archimedes wind turbine blade.

Fig. 1. Diagram of Archimedes spiral wind turbine blade.

In 2009, Timmer and Toet utilized the selective laser sinter method in order to update the Archimedes wind turbine pattern and explore the major potentials and optimum of the wind turbine power output [7]. Their finding had showed the maximum efficiency of that model was $12 \%$ while effects generated an angle of 20 degree or more were positive to its efficiency. In old HAWT scheme, lift force is the one of the main factor to take power from wind energy, but the Archimedes spiral small wind turbine is mainly depended on the drag force. In Fig. 1, a diagram of Archimedes spiral wind turbine is illustrated which can produce around $0.5 \mathrm{~kW}$ at the tip speed. The current designed wind turbine is able to be 
removing its kinetic energy by reversing wind direction. In the present research, an experimental study is accompanied to obtain evolution of the tip vortex structures in the near wake of the Archimedes wind turbine model and the aerodynamic characteristics in an open type wind tunnel by using a PIV measurement system. The Lattice Boltzmann method has been applied to investigate the behavior of aerodynamic characteristics surrounding the Archimedes wind turbine and test the capability as a design implement for the wind turbine. The focus of this research is on quantifying the evolution of the tip vortex properties and velocity distributions, including mean velocities and instantaneous velocities. This research will let us understand deeply on the turbulent wake structures.

\section{NUMERICAL}

Lattice Boltzmann Method (LBM) schemes have been intensively studied in recent years being their affinity to the computational calculation their main advantage. XFlow is one of the analysis programs for engineering analysis which it uses a proprietary, particle-based, fully Lagrangian approach by using LBM in order to simulate complex problems such as aerodynamics, aero-acoustics, moving parts and fluid-structure interaction. LBM is proposed on one distribution function which is applied for the momentum equation. Macroscopic fluid quantities such as velocity and pressure are obtained by solving the momentum equation. For the incompressible problems are computed by the following equations [8]:

$$
\Omega_{i}^{B G K}=\frac{1}{\tau}\left(f_{i}^{e q}-f_{i}\right)
$$

where the $f$ (distribution function) has been utilized to compute the velocity and density fields and $\tau$ represents the relaxation time for the flow. The $f^{e q}$ in Eq. 1 is the equilibrium distribution function for density. Usually the discretized equilibrium distribution function for D3Q19 adopts the following expression [9]:

$$
f_{i}^{e q}=\omega_{i} \rho\left[1+\frac{c_{i \alpha} u_{\alpha}}{c_{s \alpha}^{2}}+\frac{1}{2} \frac{\left(c_{i \alpha} u_{\alpha}\right)^{2}}{c_{s \alpha}^{2}}\left(\frac{c_{i \alpha} c_{i \beta}}{c_{s}^{2}}-\delta_{\alpha \beta}\right)\right]
$$

where $c_{s}$ is the sound speed, $\mathrm{u}$ the macroscopic velocity, $\delta$ the Kronecker delta, and $\omega_{i}$ are built preserving the isotropy. Fig.

2 shows that a domain established at preparation phase including flow domain in order to compute the shape of blade on XFlow. In 3D lattice Boltzmann method, the physical space is discretized into uniform Cartesian cells. Each node in the lattice units is related to its neighbors over a number of lattice velocities that are to be determined for different pattern of LBM.

In this purpose, the D3Q19 scheme has been implemented and The Wall-Adapting Local Eddy-viscosity (WALE) model has been selected which has good properties both near to and far from the wall and both for laminar and turbulent flows [10], [11]. This model recovers the asymptotic behavior of the turbulent boundary layer when this layer can be directly solved and it does not add artificial turbulent viscosity in the shear regions out of the wake. The WALE model is formulated as follows:

$$
\begin{aligned}
& v_{\text {turbulent }}=\Delta^{2} \frac{\left(G_{\alpha \beta}^{d} G_{\alpha \beta}^{d}\right)^{3 / 2}}{\left(S_{\alpha \beta} S_{\alpha \beta}\right)^{5 / 2}+\left(G_{\alpha \beta}^{d} G_{\alpha \beta}^{d}\right)^{5 / 4}} \\
& S_{\alpha \beta}=\frac{1}{2}\left(\frac{\partial v_{\alpha}}{\partial r_{\beta}}+\frac{\partial v_{\beta}}{\partial r_{\alpha}}\right) \\
& G_{\alpha \beta}^{d}=\frac{1}{2}\left(g_{\alpha \beta}^{2}+g_{\alpha \beta}^{2}\right)-\frac{1}{3} \delta_{\alpha \beta} g_{\gamma \gamma}^{2} \\
& g_{\alpha \beta}=\frac{\partial v_{\alpha}}{\partial r_{\beta}} \\
& \Delta=C_{w} V_{o l}^{1 / 3}
\end{aligned}
$$

where the WALE constant $\left(C_{w}\right)$ was set at 0.325 .

For obtaining accurate result the element number was about $3,000,000$ lattice cells on static domain which are shown in Fig. 2. In this simulation, we chose three cases to predict the aerodynamic characteristics with various inlet wind velocity which are $3.5 \mathrm{~m} / \mathrm{s}, 4 \mathrm{~m} / \mathrm{s}$ and $4.5 \mathrm{~m} / \mathrm{s}$; and rotating velocity was selected at 300rpm, 400rpm and 500rpm, respectively.

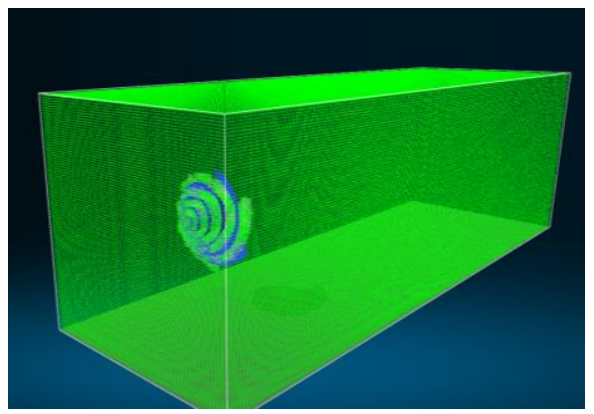

Fig. 2. Cell configuration of the wind turbine.

\section{EXPERIMENTAL}

Fig. 3 illustrates the diagram of experimental structure for PIV measurement. The 1/10 down scaled experimental model based on the Archimedes screw was engaged to gain detailed flow information. The PIV system was utilized in order to measure the $2 \mathrm{D}$ velocity component plane from horizontal stream-wise (x-y plane). The laser beam illuminated from $U$ wave mini-YAG laser was transformed into a light sheet through cylindrical and spherical lenses. The field of view for velocity field measurement is about $150 \mathrm{~mm} * 120 \mathrm{~mm}$ corresponding to the CCD resolution of $1280 * 1024$ pixels.

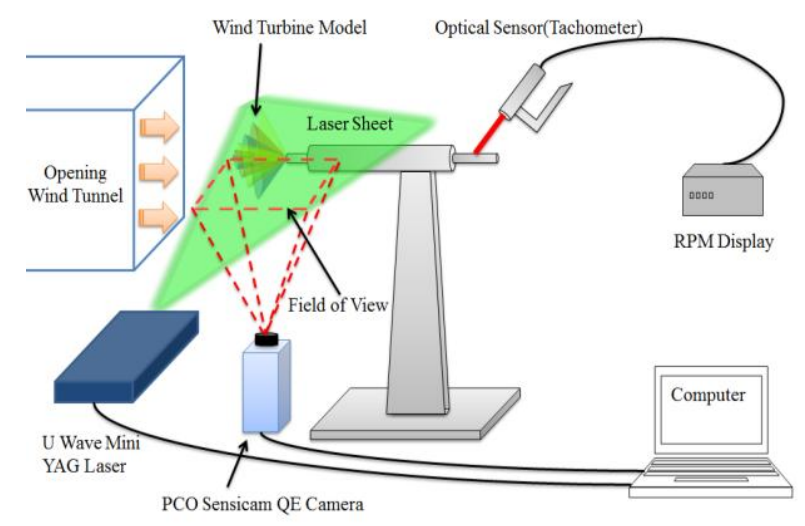

Fig. 3. Experimental setup for PIV measurement.

A digital 12bit CCD camera (PCO Sensicam qe camera) was setup vertically under the Archimedes wind turbine to 
capture particle images in $x-y$ planes. The mean velocity field was obtained by the conventional two frames cross correlation technique with 64 by 64 examination window size and $50 \%$ overlap. An entire of 2000 instantaneous velocity fields were used to provide the averaged velocity and vortical fields.

\section{RESULTS AND DISCUSSIONS}

The collective averaged velocity fields near the blade considered through PIV measurement were demonstrated in Fig. 4. The inlet air flow can be found to reduce speed significantly when it streams across the wind turbine blades. Besides that it can be seen that the speed of the incoming wind air is much higher than the local wind speed around blade's rejection. In addition to the kinetic energy of the airflow associated with the velocity deficits has been obtained by the wind turbine. There is a deformation of flow on the surface because of the tip vortices. It should be mentioned that there is a large scale vortex structure, which outcomes from a separation behind the wind blade. Regarding to the time series of instantaneous velocity fields, there are fluctuations in the tip vortices around the top of the wind turbine. These fluctuations produce a reduction of vortex intensity when the average values are calculated from the instantaneous fields. The analysis of near rotor wake shows that the blade is a major source of disturbances and unstable aerodynamic effects on account of the fact that the blade has formed as a spiral shape. The effects of the rotation speed and wind speed, which terminates the tip-speed-ratio, of the wind turbine model on the aerodynamic characteristics in the wake area were measured.

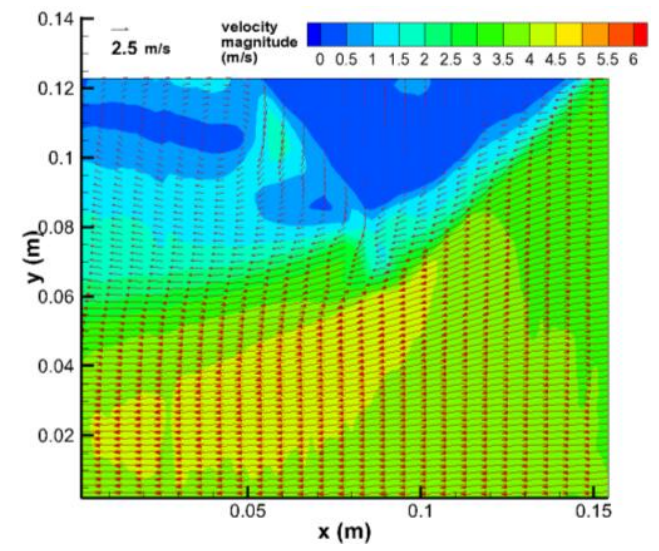

(a) $3.5 \mathrm{~m} / \mathrm{s}$ and $300 \mathrm{rpm}$.

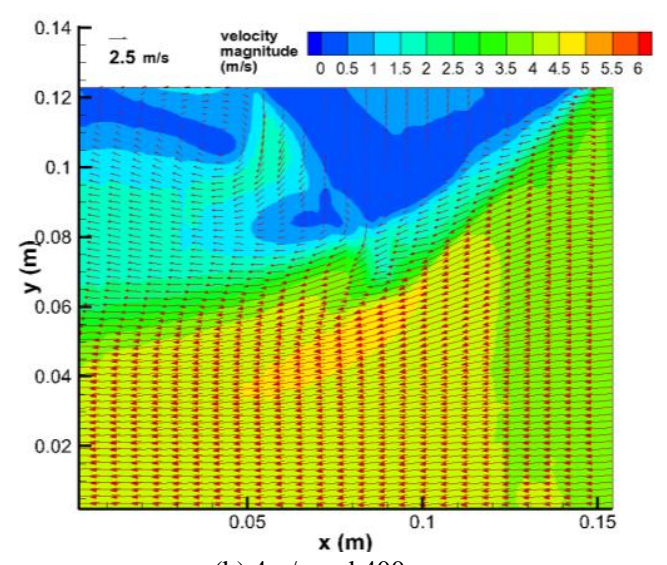

(b) $4 \mathrm{~m} / \mathrm{s}$ and $400 \mathrm{rpm}$.

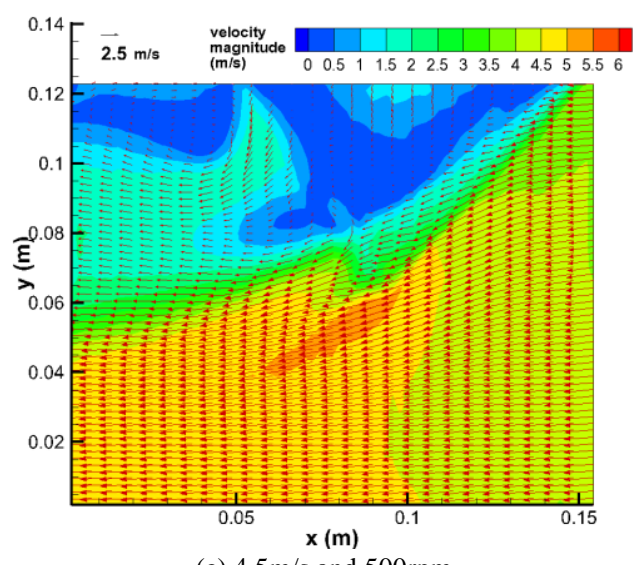

(c) $4.5 \mathrm{~m} / \mathrm{s}$ and $500 \mathrm{rpm}$

Fig. 4. Ensemble averaged velocity field obtained by PIV measurement.

The three PIV experiment results illustrate that they have a similar wake flow mark, and at the same time those contours show that the only differences were just from the comparison of the plots at different tip-speed-ratios. It is obvious that the velocity reductions in the wake region with relatively low tip-speed-ratio are shown to be smaller than that the case with relatively high tip-speed-ratio.

In order to validate the numerical results, the experimental data has been compared with the results of XFlow simulation at speed of $3.5 \mathrm{~m} / \mathrm{s}$ with angular speed of $300 \mathrm{rpm}$. Therefore, two parts of the collected region have been selected from the blade tip vortex core to the boundary as shown in Fig. 5.

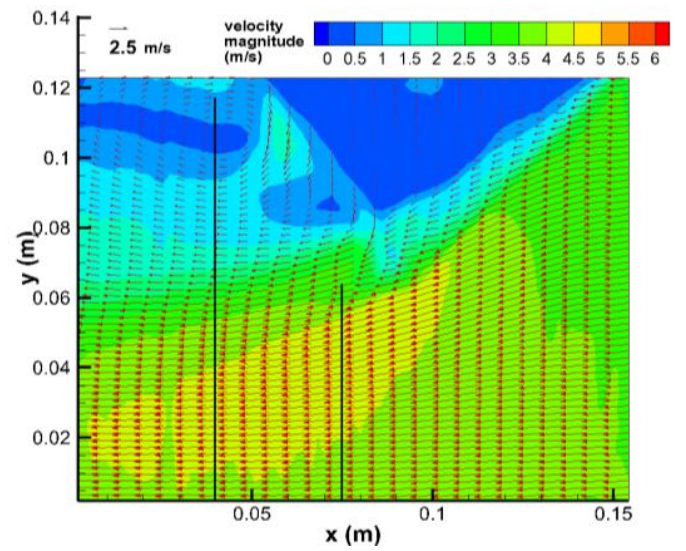

Fig. 5. Two selected lines for comparison between CFD and PIV results.

Fig. 6 indicates the comparison between numerical solution and PIV results. It seems that wind velocity of experiment is reached at the higher value than obtained numerical velocity results, and at beginning, there is a downward tendency that reflects the situation from upstream to downstream through the core of tip vortex structure.

By studying on responses on group of averaged velocity field which are gained form PIV measurement, it is found that there is a relation between lower incoming airflow and both the tip vortex structure and wake velocity. So in the spiral shaped blade, the tip vortex erection and wake velocity would be influenced much easier if we had lower incoming airflow. In addition, the influenced region is more widely than higher conditions at lower tip-speed-ratio. From these results, the flow was mainly devoted and only separated at the central of tip vortex with similar tendency circulation. It means that the downstream mean velocity value in the tip vortex of PIV is lower than the obtained tip vortex in XFlow simulation data. 


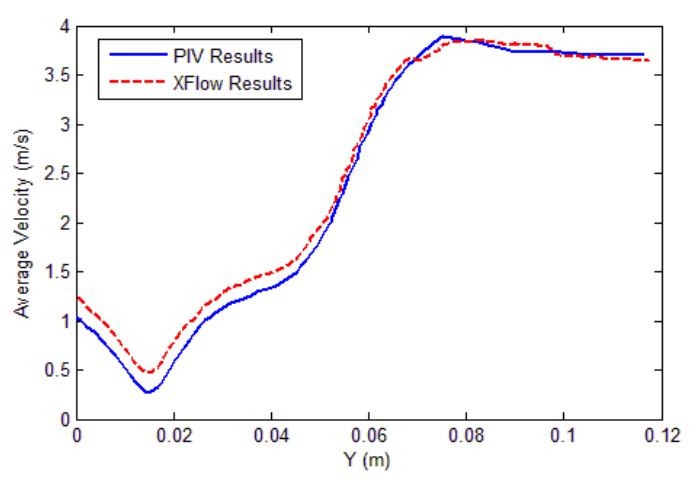

(a) Left line

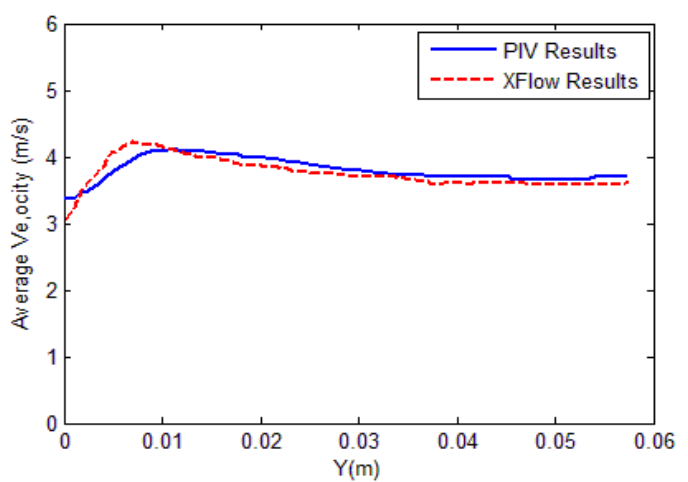

(b) Right line

Fig. 6. Comparison of experimental and numerical results.

There is a good agreement between experimental and numerical results especially around the boundary sections under 500rpm. By comparing the average velocity and pressure fields, it can be found out that the low pressure area of the suction side gradually moved to the blade edge, while the dynamic pressure is decreased at adverse static pressure, so that, the fluid flows at the blade, and before the detached vortex formed, the boundary layer would be detached. Then, the main separated vortex is increased at the further distance and the affected region is even larger. The comparison of the PIV measurement with CFD numerical simulation expresses a good agreement between the shape and the vector trend, as well as the position and size of the tip vortex.

Generally, fluid is defined base on two distribution functions, the first is the momentum equation and the energy equation is the second function in LBM. Momentum equation is normalized by characteristic length, characteristic velocity, and characteristic time. As we assume the blade and fluid around the spiral turbine have the same temperature therefore the energy term has not considered in numerical method. According to Fig. 6 the air streams through the blade and let the flow pass the blade surface with higher speed. In addition, if the air passes the wind turbine blade continuously, it would create a low-pressure area on the tip of the blade on account of the fact that the wind across this area will have higher velocity.

The tip-speed ratio, for wind turbines is illustrated in Table I. The ratio between the rotational speed of the tip of a blade and the actual velocity of the wind at speed of $5 \mathrm{~m} / \mathrm{s}$ can be reached to the maximum power at speed of 60 when the $C_{p}$ in the peak. The peak efficiency for the spiral turbine would be happened at power 1.692 when the tip-speed ratio is 2.484 . As a result, it can be seen higher tip speeds outcome require stronger blades due to large centrifugal forces.
TABLE I: EFFECTS OF DIFFERENT ANGULAR SPEED ON WIND TURBINE

\begin{tabular}{lllll}
\hline TSR & $\begin{array}{l}\text { Rotational } \\
\text { speed[rad/s }]\end{array}$ & $\begin{array}{l}\text { Torque } \\
{[\mathrm{Nm}]}\end{array}$ & Power & $C p$ \\
\hline 0 & 0 & 0.0964 & 0 & 0.000 \\
\hline 0.414 & 10 & 0.063 & 0.63 & 0.063 \\
\hline 0.828 & 20 & 0.0571 & 1.142 & 0.115 \\
\hline 1.242 & 30 & 0.048 & 1.44 & 0.144 \\
\hline 1.656 & 40 & 0.0395 & 1.58 & 0.158 \\
\hline 2.07 & 50 & 0.0338 & 1.69 & 0.170 \\
\hline 2.484 & 60 & 0.0282 & 1.692 & 0.170 \\
\hline 2.898 & 70 & 0.02 & 1.4 & 0.140 \\
\hline 3.312 & 80 & 0.0116 & 0.928 & 0.093 \\
\hline 3.726 & 90 & 0.002 & 0.18 & 0.018 \\
\hline 4.14 & 100 & -0.00416 & -0.416 & -0.042 \\
\hline
\end{tabular}

Fig. 7 illustrates a comparison of moment of force in the range of rotational speed from 1 to $120 \mathrm{rad} / \mathrm{s}$ for different inlet velocities. As can be seen from Fig. 7, at first there is a positive correlation between rotational speed and torque until the maximum torque occurs, then the moment of the blade is gradually declining when the angular speed is rising for all different velocity of air; $7 \mathrm{~m} / \mathrm{s}, 6 \mathrm{~m} / \mathrm{s}$ and $5 \mathrm{~m} / \mathrm{s}$. In addition, from the figure if the speed of air increased the tendency of a force to rotate the blade about an $\mathrm{x}$ axis would be increase which lead to revolve the blade faster with higher power.

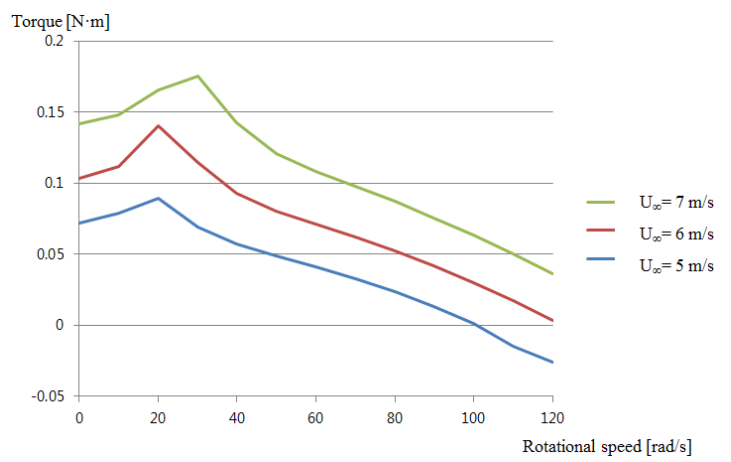

Fig. 7. Comparison of torque profiles between three different inlet air velocities.

Fig. 8 presents the value of $C_{p}$ against the tip speed ratio rate with regards to the various rage of the air velocity from 5 $\mathrm{m} / \mathrm{s}$ to $12 \mathrm{~m} / \mathrm{s}$. The maximum power coefficient can be observed near the tip speed ratio is about 2.5 for all inlet air speed and has approximately 0.25 . The power coefficient rates have the same tendency regardless of air velocities. The modern urban usage 3-blade and Darrieus blade type wind turbines have high rotor efficiency at high tip speed ratio range. It means that high efficiency can be generated in case of high wind speed. Compared to the aerodynamic performance of the other blades in the lower tip speed ratio range, the spiral wind blade with Archimedes shape shows relatively high rotor efficiency.

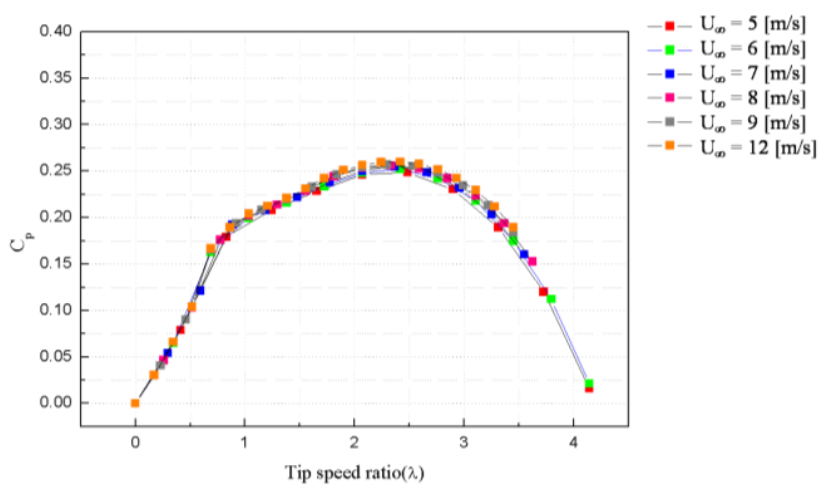

Fig. 8. $C p$-Tip speed ratio with respect to the wind velocity. 
In addition, the blade had high $C_{p}$ values in a wider range of the tip speed ratio. Regarding to the total aerodynamic characteristics, it can be concluded that the Archimedes spiral turbine is appropriate design for using urban wind.

\section{CONCLUSION}

In this study, the PIV technique and numerical methods were performed to study the behavior of the flow around the turbine and efficiency power near the wake of the Archimedes spiral wind turbine blade. As results, our gained finding can be summarized as follows:

1) The rotational speed of the blade is directly influenced base on the speed of inlet air. In addition, our investigation demonstrated the blade can be reach at angular speed around 500rpm while the inlet velocity is $4.5 \mathrm{~m} / \mathrm{s}$.

2) The inner low speed section rotated the same turning direction of the blade. The relative velocity of the flow is closed zero to the blade.

3) The investigation of the obtained results determined the straight interaction between the mean flow at the rotor downstream and the induced velocity due to the tip vortices.

4) The finding exposed the presence of important vortex constructions downstream the hub and near the root of the blade. Some unpredictability of the helical tip vortices is also distinguished. Because of these fluctuations, the instantaneous velocity field is very rich with the evidences.

5) The maximum efficiency for the spiral turbine would be happened when the $C_{p}$ is in the peak.

6) Numerical simulation results have a good agreement with the PIV experiments.

7) Regarding to the PIV experiment, the provided information of the blade flow for different wind velocity of attack are helpful to improve the performance and the design of Archimedes spiral wind turbine.

\section{REFERENCES}

[1] Small Wind World Report Summary 2012, World Wind Energy Association, 2012
[2] A. A. Edward and B. W. Philip, "The effect of wind on energy consumption in buildings," Energy and Buildings, vol. 1, no. 1, pp. 77-84, 1977.

[3] Renewable Energy Policy Network for the 21st Century, Renewables 2011 Global Status Report, 2011

[4] H. Cao, "Aerodynamics analysis of small horizontal axis wind turbine blades by using 2D and 3D CFD modeling", M.S. thesis, University of the Central Lancashire, 2011.

[5] N. J. Choi, S. H. Nam, J. H. Jeong, and K. C. Kim, "Numerical study on the horizontal axis turbines arrangement in a wind farm: Effect of separation distance on the turbine aerodynamic power output," Journal of Wind Engineering and Industrial Aerodynamics, vol. 117, pp. 11-17, 2013.

[6] W. A. Timmer and S. Toet, "Verslag van de metingen aan de archimedes in de lage-snelheids windtunnel van dnw". TU Delft, 2009.

[7] Q. Lu, Q. Li, Y. K. Kim and K. C. Kim, "A study on design and aerodynamic characteristics of a spiral-type wind turbine blade," Journal of KSV, vol. 10, no. 1, pp. 27-33, 2012.

[8] Y. H. Qian, D. d'Humieres, and P. Lallemand, "Lattice BGK models for NavierStokes equation," EPL (Europhysics Letters), vol. 17, no. 6, pp. 479, 1992.

[9] C. S. Michael and T. T. Jr. Daniel, Lattice Boltzmann Modeling: An Introduction for Geoscientists and Engineer, $1^{\text {st }}$ ed. Spring, 2006, ch. 4, pp. 34-54.

[10] M. Weickert, G. Teike, O. Schmidt, and M. Sommerfeld, "Investigation of the LES WALE turbulence model within the lattice Boltzmann framework," Computers \& Mathematics with Applications, vol. 5, pp. 2200-2214, 2010.

[11] C. S. Zhuo and C. W. Zhong, "LES-based filter-matrix lattice Boltzmann model for simulating turbulent natural convection in a square cavity," International Journal of Heat and Fluid Flow, vol. 42, pp. 10-22, 2013

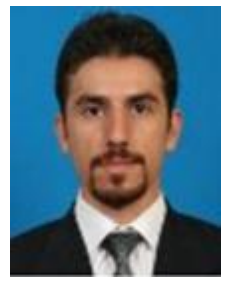

Arman Safdari received master degree in mechanical engineering from Technical University of Malaysia in 2012. Currently he is a postgraduate student at the Faculty of Mechanical Engineering, Pusan National University.

His current research interests include simulation and modeling of fluid flow, flow structure interaction, renewable Energy, heat transfer, numerical methods and advanced engineering mathematics theory.

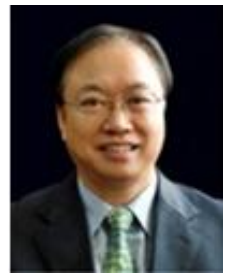

Kyung Chun Kim is a professor in the School of Mechanical Engineering of Pusan National University. He obtained his Ph.D. degree from the Korea Advanced Institute of Science and Technology (KAIST), Korea, in 1987.

His research interests include multi-scale multi-physics measurements, such as micro-PIV/LIF, bio-MEMS, design and fabrication of thermal management system, organic Rankine cycle, renewable energy, etc. 